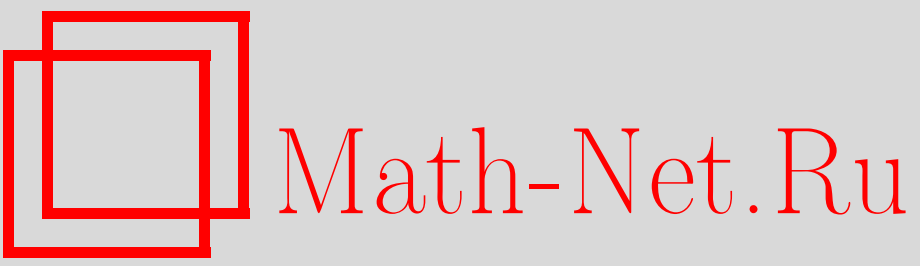

А. И. Вдовин, Д. С. Косов, В. Навроцка, Самосогласованное приближение случайных фаз в горячих конечных ферми-системах, ТМФ, 1997, том 111, номер 2, 279-288

DOI: https://doi.org/10.4213/tmf1007

Использование Общероссийского математического портала Math-Net.Ru подразумевает, что вы прочитали и согласны с пользовательским соглашением

http://www. mathnet.ru/rus/agreement

Параметры загрузки :

IP : 54.174 .149 .18

26 апреля 2023 г., 13:39:36 
ТЕОРЕТИЧЕСКАЯ

И МАТЕМАТИЧЕСКАЯ

ФИЗИКА

Том 111, № 2

май, 1997

\author{
А. И. В довин* ${ }^{*}$ Д. С. Косов* , В. Навроцка ${ }^{\dagger}$
}

\title{
САМОСОГЛАСОВАННОЕ ПРИБЛИЖЕНИЕ СЛУЧАЙНЫХ ФАЗ В ГОРЯЧИХ КОНЕЧНЫХ ФЕРМИ-СИСТЕМАХ
}

\begin{abstract}
Для изучения коллективных возбуждений в нагретых системах конечного числа фермионов (атомных ядрах, металлических кластерах) с помощью формализма термополевой динамики разработан метод, который учитывает влияние на среднее поле температуры системы и параметров, определяющих коллективные возбуждения системы. Более конкретно, чем в обычном тепловом приближении случайных фаз, учтена роль принципа Паули.
\end{abstract}

\section{1. ВВЕДЕНИЕ}

В последние годы усилился интерес к системам конечного числа фермионов, что связано, в частности, с увеличением разнообразия таких систем, создаваемых и исследуемых в лабораторных условиях. Помимо таких давно известных объектов, как атомные ядра, сейчас интенсивно изучаются двумерные системы из нескольких электронов (так называемые квантовые точки) и кластеры, образованные конечным числом атомов или молекул.

По крайней мере некоторые из перечисленных ферми-систем долго живут и в сильно возбужденном состоянии, а, значит, можно экспериментально исследовать эволюцию их свойств в зависимости от температуры. Довольно много известно о поведении так называемых горячих (с энергией возбуждения $E_{x} \sim 100-300$ МэВ) атомных ядер - об изменении их формы, коллективных колебаниях. Специфика экспериментов с металлическими кластерами такова, что исследуются именно нагретые кластеры.

Основная проблема теоретического изучения сильно возбужденных систем заключается в том, что плотность уровней в них чрезвычайно высока. "Квазиконтинуум" уровней делает невозможным реалистические расчеты в микроканоническом ансамбле и вынуждает прибегать к статистическому описанию, которое в той или иной мере опирается на методы квантовой теории поля при конечных температурах. Выбор конкретного квантово-полевого метода зависит от вида решаемой задачи.

* Объединенный институт ядерных исследований, Лаборатория теоретической физики им. Н. Н. Боголюбова, Дубна, Россия

${ }^{\dagger}$ Институт теоретической физики Вроцлавского университета, Польша 
Мы рассмотрим коллективное движение сильно возбужденной конечной ферми-системы, относительно которой предполагается, что она удерживается в связанном состоянии благодаря двухчастичному (быть может, эффективному) взаимодействию составляюших ее частиц. Кроме того, характер этого взаимодействия допускает сушествование в системе общего для всех частиц среднего поля. Предлагается новый подход, который, во-первых, принимает во внимание влияние на среднее поле температуры $T$ и параметров, определяющих коллективные возбуждения системы, а во-вторых, более корректно, чем это присуше обычному тепловому приближению случайных фаз (ТПСФ), учитьвает принцип Паули. Мы используем формализм термополевой динамики (ТПД) [1], который нам представляется наиболее подходящим для данной задачи.

\section{2. ТЕРМОПОЛЕВАЯ ДИНАМИКА: КРАТКОЕ ИЗЛОЖКНИЕ ФОРМАЛИЗМА}

Формализм ТПД используется нами в том виде, как это было сделано в работах [2-5] для случая сильно возбужденных (горячих) ядер. Предположим, что нагретая система конечного числа фермионов в состоянии теплового равновесия описьвается функцией распределения большого канонического ансамбля. Основная идея ТП Д-построить для $T \neq 0$ формализм, обладаюший преимушествами квантовой теории поля, и такой, в частности, чтобы среднее по ансамблю от некоторого оператора $A$ при $T \neq 0$

$$
\langle\langle A\rangle\rangle=\frac{1}{\operatorname{Tr}(\exp (-H / T))} \operatorname{Tr}[A \exp (-H / T)]
$$

можно было вычислить как среднее по некоторому зависящему от $T$ состоянию $|0(T)\rangle$, которое в дальнейшем мы будем называть тепловым вакуумом, т.е.

$$
\langle\langle A\rangle\rangle=\langle 0(T)|A| 0(T)\rangle
$$

здесь $H$ - гамильтониан системы, собственные состояния которого - $|n\rangle$, а соответствующие собственные значения $-E_{n}$.

Сформулированная выше цель достигается в ТПД формальным удвоением числа степеней свободы системы - введением "тильда-состояний" $|\tilde{n}\rangle$. "Тильда-состояния" - собственные состояния "тильда-гамильтониана" $\widetilde{H}$ с теми же собственными значениями, что и исходные состояния $|n\rangle$, т.е. $\widetilde{H}|\tilde{n}\rangle=E_{n}|\tilde{n}\rangle$. Любой оператор $A$, действуюший в пространстве обычных состояний $|n\rangle$, имеет свой “тильда-образ”, действующий в пространстве состояний $|\tilde{n}\rangle$. Операция “тильда" задается следуюшими правилами:

$$
(A B)^{\sim}=\tilde{A} \widetilde{B}, \quad(a A+b B)^{\sim}=a^{*} \tilde{A}+b^{*} \widetilde{B},
$$

где $a, b-c$-числа, а звездочка означает комплексное сопряжение. Операция "тильда" коммутирует с операцией эрмитова сопряжения. Обычные и “тильда-операторы" коммутируют или антикоммутируют друг с другом в зависимости от того, являются ли они бозонными или фермионными операторами. Двукратное применение операции “тильда" к одному и тому же оператору дает следующий результат: $(\tilde{\tilde{A}})= \pm A$ (знак $(-)$ отвечает фермионному оператору $A$, а знак $(+)$ - бозонному). 
Прямое произведение пространств обычных и “тильда-состояний” и составляет полное гильбертово пространство состояний нагретой системы. В этом пространстве оператор сдвига по оси времени - тепловой гамильтониан $\mathcal{H}=H-\widetilde{H}$. Это означает, что для изучения возбужденных состояний нагретой системы нужно диагонализовать $\mathcal{H}$.

\section{3. ПРИБЛИЖЕНИЕ СРЕДНЕГО ПОЛЯ В ТЕРМОПОЛЕВОЙ ДИНАМИКЕ}

Рассмотрим находящуюся в термодинамическом равновесии систему $N$ частищ с гамильтонианом, состояшим из кинетической энергии и двухчастичного взаимодействия:

$$
H=\sum_{12} t_{12} a_{1}^{+} a_{2}+\frac{1}{4} \sum_{1234} V_{1234} a_{1}^{+} a_{2}^{+} a_{4} a_{3},
$$

где $a^{+}, a$ - операторы рождения и уничтожения фермионов,

$$
t_{12}=T_{12}-\lambda \delta_{12}
$$

$\lambda$ - химический потенциал, $T_{12}$ - матрица кинетической энергии. Использование в ТПД усреднения по векторам из гильбертова пространства вместо усреднения по статистическому ансамблю позволяет представить гамильтониан (2) в нормальной форме, связанной с зависяшим от температуры тепловым основным состоянием $\left|\Psi_{0}(T)\right\rangle$. Волновая функция $\left|\Psi_{0}(T)\right\rangle$ - это решение задачи на собственные значения [1]:

$$
\mathcal{H}\left|\Psi_{0}(T)\right\rangle=(H-\widetilde{H})\left|\Psi_{0}(T)\right\rangle=0
$$

При помощи теоремы Вика для одно- и двухчастичных операторов получаются следуюшие выражения:

$$
\begin{gathered}
a_{i}^{+} a_{j}=\rho_{j i}+: a_{i}^{+} a_{j}: \\
a_{1}^{+} a_{2}^{+} a_{4} a_{3}= \\
\rho_{31} \rho_{42}-\rho_{41} \rho_{32}-\rho_{41}: a_{2}^{+} a_{3}:+ \\
+\rho_{31}: a_{2}^{+} a_{4}:+\rho_{42}: a_{1}^{+} a_{3}:- \\
-\rho_{32}: a_{1}^{+} a_{4}:+: a_{1}^{+} a_{2}^{+} a_{4} a_{3}:
\end{gathered}
$$

здесь

$$
\rho_{i j}=\left\langle a_{j}^{+} a_{i}\right\rangle
$$

- одночастичная матрица плотности, а $\langle\ldots\rangle$ означает статистическое среднее (т.е. матричный элемент по тепловому основному состоянию $\left.\left|\Psi_{0}(T)\right\rangle\right)$.

Представим исходньй гамильтониан в следуюшем виде:

$$
H=h_{0}+h_{11}+h_{22} \text {, }
$$


где

$$
\begin{aligned}
h_{0} & =\sum_{12} t_{12} \rho_{21}+\frac{1}{2} \sum_{1234} V_{1234} \rho_{31} \rho_{42}, \\
h_{11} & =: \sum_{12} t_{12} a_{1}^{+} a_{2}+\sum_{1234} \rho_{42} a_{1}^{+} a_{3}: \\
h_{22} & =: \frac{1}{4} \sum_{1234} V_{1234} a_{1}^{+} a_{2}^{+} a_{4} a_{3}:
\end{aligned}
$$

Слагаемое $h_{11}$ - квадратичная форма по фермионным операторам $a^{+}, a$. Подобные квадратичные формы могут быть точно диагонализованы при помоши унитарного преобразования [6]:

$$
a_{l}^{+}=\sum_{k} D_{l k}^{*} \alpha_{k}^{+}, \quad a_{l}=\sum_{k} D_{l k} \alpha_{k}
$$

где $D D^{+}=D^{+} D=I$. Операторы $\alpha_{k}^{+}, \alpha_{k}$ - операторы рождения и уничтожения новых фермионов (квазичастиц).

Условие диагональности $h_{11}$ приводит к следуюшей системе уравнений для матричных элементов матришы $D$ :

$$
\sum_{2}\left(t_{12}+\sum_{34} V_{1234} \rho_{43}\right) D_{25}=\varepsilon_{5} D_{15}
$$

которая должна быть дополнена условием сохранения среднего числа частиц

$$
\sum_{1} \rho_{11}=N
$$

Если отбросить слагаемое $h_{22}$, то оставшаяся часть гамильтониана $H$ диагональна относительно операторов квазичастиц $\alpha_{k}^{+}, \alpha_{k}$. Это стандартное приближение Хартри-Фока с зависимостью от температуры. Величины $\varepsilon_{i}$ в системе уравнений (4) - энергии квазичастиц.

В таком подходе температура введена в теорию с помошью волновой функции основного состояния $\left|\Psi_{0}(T)\right\rangle$, которую в приближении Хартри-Фока следует отождествить с тепловым вакуумом ТПД $|0(T)\rangle[1-5]$. Действительно, оказывается, что функция $|0(T)\rangle$ - вакуум для операторов уничтожения новых "тепловых" квазичастиц ( и их “тильда-образов") $\beta, \tilde{\beta}$ :

$$
\beta_{i}|0(T)\rangle=\tilde{\beta}_{i}|0(T)\rangle=0 .
$$

Тепловые квазичастицы связаны с хартри-фоковскими квазичастищами $\alpha_{k}^{+}, \alpha_{k}$ и их “тильда-образами" следующим унитарным преобразованием [1]:

$$
\alpha_{i}=x_{i} \beta_{i}+y_{i} \tilde{\beta}_{i}^{+}, \quad \alpha_{i}^{+}=x_{i} \beta_{i}^{+}+y_{i} \tilde{\beta}_{i}
$$

где

$$
x_{i}=\sqrt{1-n_{i}}, \quad y_{i}=\sqrt{n_{i}}, \quad n_{i}=\frac{1}{1+\exp \left(\frac{\varepsilon_{i}}{T}\right)} .
$$


Среднее значение некоторого оператора $A$ по тепловому вакууму $|0(T)\rangle$ совпадает со средним значением этого оператора по большому каноническому ансамблю с гамильтонианом Хартри-Фока (см. раздел 1). Тепловой гамильтониан системы в приближении Хартри-Фока

$$
\mathcal{H}=\sum_{1} \varepsilon_{1}\left(\alpha_{1}^{+} \alpha_{1}-\tilde{\alpha}_{1}^{+} \tilde{\alpha}_{1}\right)
$$

остается диагональным и после преобразования (6):

$$
\mathcal{H}=\sum_{1} \varepsilon_{1}\left(\beta_{1}^{+} \beta_{1}-\tilde{\beta}_{1}^{+} \tilde{\beta}_{1}\right) .
$$

В этом же приближении для одночастичной матрицы плотности справедливо выражение

$$
\rho_{12}=\left\langle 0(T)\left|a_{2}^{+} a_{1}\right| 0(T)\right\rangle=\sum_{k} D_{2 k}^{*} D_{1 k} n_{k} .
$$

\section{4. УРАВНЕНИЯ САМОСОГЛАСОВАННОГО ТЕПЛОВОГО ПРИБЛИЖЕНИЯ СЛУЧАЙНЫХ ФАЗ}

Перейдем к обсуждению роли взаимодействия хартри-фоковских квазичастиц. Выраженное через операторы $\alpha_{k}^{+}, \alpha_{k}$ взаимодействие $h_{22}$ имеет вид

$$
h_{22}=: \frac{1}{4} \sum_{1234} U_{1234} \alpha_{1}^{+} \alpha_{2}^{+} \alpha_{4} \alpha_{3}:
$$

где

$$
U_{i j k l}=\sum_{1234} V_{1234} D_{1 i}^{*} D_{2 j}^{*} D_{3 k} D_{4 l}
$$

Тем самым полный тепловой гамильтониан системы выплядит следуюшим образом:

$$
\mathcal{H}=: \sum_{1} \varepsilon_{1}\left(\alpha_{1}^{+} \alpha_{1}-\tilde{\alpha}_{1}^{+} \tilde{\alpha}_{1}\right)+\frac{1}{4} \sum_{1234} U_{1234}\left(\alpha_{1}^{+} \alpha_{2}^{+} \alpha_{4} \alpha_{3}-\tilde{\alpha}_{1}^{+} \tilde{\alpha}_{2}^{+} \tilde{\alpha}_{4} \tilde{\alpha}_{3}\right):
$$

Чтобы найти спектр возбуждений горячей системы, следует записать гамильтониан (9) через операторы тепловых квазичастищ (6), как это было сделано выше для его хартри-фоковской части. В дальнейшем мы будем оперировать только с той частью взаимодействия $h_{22}$, которую учитывают в ТПСФ (см. приложение, формула (П.1)). В этом приближении сама структура $\mathcal{H}$ подсказывает нам, какие комбинации операторов тепловых квазичастиц можно использовать в качестве “строительных блоков" для конструирования волновой функции возбужденного состояния. Именно для этой цели следует взять бифермионные операторы

$$
A_{12}^{+}=\beta_{1}^{+} \tilde{\beta}_{2}^{+}, \quad A_{12}=\tilde{\beta}_{2} \beta_{1} .
$$

Точньй коммутатор этих операторов имеет вид

$$
\left[A_{12}, A_{34}^{+}\right]=\delta_{13} \delta_{24}-\delta_{13} \tilde{\beta}_{4}^{+} \tilde{\beta}_{2}-\delta_{24} \beta_{3}^{+} \beta_{1} .
$$


Стандартное приближение ТПСФ состоит в том, что в правой части коммутатора оставляют только $\delta$-символы, т.е. бифермионные операторы рассматриваются как бозонные. Мы сделаем шаг дальше и будем использовать для коммутатора (10) следующее выражение $[7,8]$ :

$$
\left[A_{12}, A_{34}^{+}\right]=\delta_{13} \delta_{24}\left(1-q_{1}-q_{2}\right)
$$

где $q_{i}-c$-числа:

$$
\left\langle\beta_{1}^{+} \beta_{2}\right\rangle=\left\langle\tilde{\beta}_{1}^{+} \tilde{\beta}_{2}\right\rangle=\delta_{12} q_{1}
$$

В (11) среднее вычисляется уже не по хартри-фоковскому тепловому вакууму $|0(T)\rangle$ (такое среднее равно нулю), а по новому тепловому основному состоянию системы, которое будет определено ниже.

Подстановкой $[7,8]$

$$
b_{12}=\frac{A_{12}}{\sqrt{1-q_{12}}}, \quad b_{12}^{+}=\frac{A_{12}^{+}}{\sqrt{1-q_{12}}},
$$

где $q_{12}=q_{1}+q_{2}$, бифермионные операторы, удовлетворяющие коммутационному соотношению (11), снова, как в ТПСФ, преврашаются в бозонные:

$$
\left[b_{12}, b_{34}^{+}\right]=\delta_{13} \delta_{24}
$$

Гармоническая часть теплового гамильтониана $\mathcal{H}$ - квадратичная форма по новым бозонным операторам $b_{i k}^{+}, b_{i k}$ (см. (П.2)) и может быть точно диагонализована при помощи преобразования Боголюбова [6]:

$$
\begin{aligned}
& b_{12}^{+}=\sum_{\nu} \psi_{12}^{\nu} Q_{\nu}^{+}+\phi_{12}^{\nu} Q_{\nu}, \\
& b_{12}=\sum_{\nu} \psi_{12}^{\nu} Q_{\nu}+\phi_{12}^{\nu} Q_{\nu}^{+},
\end{aligned}
$$

где амплитуды $\psi_{12}^{\nu}$ и $\phi_{12}^{\mu}$ удовлетворяют следующему условию:

$$
\sum_{12} \psi_{12}^{\nu} \psi_{12}^{\mu}-\phi_{12}^{\nu} \phi_{12}^{\mu}=\delta_{\nu \mu} .
$$

Поскольку операторы $Q_{\nu}^{+}, Q_{\nu}$ (будем называть их операторами фононов) диагонализуют гамильтониан (П.2) и он приобретает вид гамильтониана системы невзаимодействующих осцилляторов с частотами $\omega_{\nu}$, волновые функции возбужденных состояний системы будут иметь вид $\left|\Psi_{\nu}(T)\right\rangle=Q_{\nu}^{+}\left|\Psi_{0}(T)\right\rangle$, где $\left|\Psi_{0}(T)\right\rangle$ - тепловой фононньй вакуум:

$$
Q_{\nu}\left|\Psi_{0}(T)\right\rangle=0
$$

Очевидно, что фононный вакуум является основным состоянием гармонического гамильтониана (П.2) с нулевой энергией. Числа заполнения тепловых квазичастиц $q_{i}$ вычисляются усреднением оператора $\beta_{i}^{+} \beta_{i}$ именно по этому состоянию.

Уравнения для амплитуд $\psi_{12}^{\nu}, \phi_{12}^{\nu}$ и частот $\omega_{\nu}$ колебаний системы могут быть получены с помощью уравнений движения. Поскольку

$$
\left[\mathcal{H}, Q_{\nu}^{+}\right]=\omega_{\nu} Q_{\nu}^{+}
$$


то имеем

$$
\begin{aligned}
\varepsilon_{24} \psi_{24}^{\nu} & +\frac{1}{2} \sum_{13} U_{1234} \sqrt{1-q_{13}} \sqrt{1-q_{24}} x_{2} y_{4}\left(x_{1} y_{3} \phi_{13}^{\nu}+y_{1} x_{3} \psi_{31}^{\nu}\right)- \\
& -\frac{1}{2} \sum_{13} U_{1234} \sqrt{1-q_{13}} \sqrt{1-q_{24}} x_{4} y_{2}\left(x_{3} y_{1} \phi_{13}^{\nu}+y_{3} x_{1} \psi_{31}^{\nu}\right)=\omega_{\nu} \psi_{24}^{\nu} \\
\varepsilon_{42} \phi_{42}^{\nu} & +\frac{1}{2} \sum_{13} U_{1234} \sqrt{1-q_{13}} \sqrt{1-q_{24}} x_{4} y_{2}\left(x_{1} y_{3} \phi_{13}^{\nu}+y_{1} x_{3} \psi_{31}^{\nu}\right)- \\
& -\frac{1}{2} \sum_{13} U_{1234} \sqrt{1-q_{13}} \sqrt{1-q_{24}} x_{2} y_{4}\left(x_{3} y_{1} \phi_{13}^{\nu}+y_{3} x_{1} \psi_{31}^{\nu}\right)=-\omega_{\nu} \phi_{42}^{\nu}
\end{aligned}
$$

Заменив переменные $\psi_{24}^{\nu}, \phi_{42}^{\nu}$ их линейными комбинациями

$$
X_{24}^{\nu}=\frac{1}{x_{2} y_{4}+y_{2} x_{4}}\left(\psi_{24}^{\nu}-\phi_{42}^{\nu}\right), \quad Y_{24}^{\nu}=\frac{1}{x_{2} y_{4}-y_{2} x_{4}}\left(\psi_{24}^{\nu}+\phi_{42}^{\nu}\right),
$$

мы получаем для переменных $X_{24}^{\nu}, Y_{24}^{\nu}$ две независимые системы уравнений:

$$
\begin{gathered}
\varepsilon_{24} X_{24}^{\nu}+\frac{1}{2} \sum_{13} U_{1234} \sqrt{1-q_{13}} \sqrt{1-q_{24}}\left(n_{1}-n_{3}\right) X_{31}^{\nu}=\omega_{\nu} X_{24}^{\nu} \\
\varepsilon_{24} Y_{24}^{\nu}+\frac{1}{2} \sum_{13} U_{1234} \sqrt{1-q_{13}} \sqrt{1-q_{24}}\left(n_{1}-n_{3}\right) Y_{31}^{\nu}=\omega_{\nu} Y_{24}^{\nu}
\end{gathered}
$$

причем имеет место соотношение

$$
X_{24}^{\nu}=\frac{\omega_{\nu}}{\left|\omega_{\nu}\right|} Y_{24}^{\nu}
$$

Система уравнений (13) связана с системой уравнений для термодинамически равновесного среднего поля (4), (5) через одночастичную матрицу плотности $\rho_{i j}(3)$. В качестве теплового основного состояния, по которому проводится усреднение в (3), теперь следует взять фононный вакуум $\left|\Psi_{0}(T)\right\rangle$ :

$$
\rho_{12}=\left\langle\Psi_{0}(T)\left|a_{2}^{+} a_{1}\right| \Psi_{0}(T)\right\rangle=\sum_{k} D_{2 k}^{*} D_{1 k}\left(x_{k}^{2} q_{k}+y_{k}^{2}\left(1-q_{k}\right)\right) .
$$

Квазичастичные числа заполнения $q_{i}$ следующим образом выражаются через амплитуды фононных волновых функций и тепловые числа заполнения хартри-фоковских состояний [9]:

$$
q_{i}=\sum_{\substack{\nu>0 \\ k}}\left[\left(X_{i k}^{\nu} x_{k} y_{i}\right)^{2}+\left(X_{k i}^{\nu} y_{k} x_{i}\right)^{2}\right]
$$

здесь $\nu>0$ означает, что суммирование проводится только по положительным решениям системы (13). 
Выпишем теперь полную систему уравнений самосогласованного температурного приближения случайных фаз, объединив уравнения (4), (5) и уравнения (13)-(15):

$$
\begin{gathered}
\sum_{2}\left[t_{12}+\sum_{345} V_{1234} D_{26}^{*} D_{46}\left(x_{5}^{2} q_{5}+y_{5}^{2}\left(1-q_{5}\right)\right)\right] D_{26}=\varepsilon_{6} D_{16} \\
\sum_{12} D_{12}^{*} D_{12}\left(x_{2}^{2} q_{2}+y_{2}^{2}\left(1-q_{2}\right)\right)=N \\
\varepsilon_{24} X_{24}^{\nu}+\frac{1}{2} \sum_{13} \sum_{5678}\left(V_{5678} D_{51}^{*} D_{62}^{*} D_{73} D_{84}\right) \times \\
\times \sqrt{1-q_{13}} \sqrt{1-q_{24}}\left(n_{1}-n_{3}\right) X_{31}^{\nu}=\omega_{\nu} X_{24}^{\nu} \\
q_{1}=\sum_{\nu>0}\left[\left(X_{12}^{\nu} x_{2} y_{1}\right)^{2}+\left(X_{21}^{\nu} y_{2} x_{1}\right)^{2}\right]
\end{gathered}
$$

Эта система нелинейных уравнений с очевидностью демонстрирует не только зависимость среднего поля (другими словами, одночастичного движения) и параметров коллективных возбуждений от температуры, но и зависимость одночастичного движения от коллективного. Возникновение подобной зависимости представляется нетривиальным. Оно непосредственно связано с тем, что мы учли отличие основного состояния системы от теплового хартри-фоковского вакуума и наличие в этом основном состоянии виртуальных возбуждений тепловых квазичастиц. Учет таких виртуальных возбуждений приводит к появлению в уравнениях для коллективных амплитуд множителей $\sqrt{1-q_{13}}, \sqrt{1-q_{24}}$. Хотя и в обычном ТПС $\Phi$ основное состояние, строго говоря, не совпадает с хартри-фоковским, ТПС $\Phi$-корреляции в основном состоянии недостаточны, для того чтобы возникла обратная связь между коллективными колебаниями и средним полем, т.к. в этом приближении $q_{i}=0$. Как легко видеть, если в вышеприведенной системе сделать эту стандартную для ТПСФ подстановку (т.е. $q_{i}=0$ ), то уравнения $\left(4^{\prime}\right),\left(5^{\prime}\right)$ замыкаются друг на друга, а их решение уже в качестве внешнего параметра входит в уравнение для коллективных возбуждений $\left(13^{\prime}\right)$, которое просто совпадает с уравнением ТПСФ.

\section{5. ЗАКЛЮЧЕНИЕ}

Мы получили систему уравнений, согласованно описывающую одночастичное и коллективное движение в сильно возбужденной (нагретой) системе конечного числа фермионов. В нашем подходе коллективное движение в системе рассматривается более последовательно, чем в ТПСФ, в частности, учитывается влияние принципа Паули на фермионную структуру коллективных колебаний. Это приводит к возникновению зависимости среднего поля системы от параметров коллективного движения, хотя последнее, как и в ТПСФ, предполагается гармоническим. Предложенный нами метод открывает новые перспективы в изучении коллективного движения большой амплитуды в сильно возбужденных (нагретых) ядрах. В температурном самосогласованном приближении случайных фаз влияние сложных многоквазичастичных конфигураций учитывается уже при построении среднего поля, т.к. принимается во внимание влияние остаточного взаимодействия хартри-фоковских квазичастищ на одночастичную матрицу плотности. 
Авторы благодарны А. В. Авдеенкову за полезные обсуждения.

Настолшая работа выполнена при частичной поддержке Российского фонда фундаментальных исследований (грант 95-02-05701) и гранта Республики Польша KBN № 2 Р 302 01804. Один из авторов (Д. С.К.) признателен за гостеприимство Институту теоретической физики Вроцлавского университета.

\section{ПРИЛОЖЕНИЕ}

Здесь мы приведем выражения для гармонической части теплового гамильтониана в терминах операторов тепловых квазичастищ $\beta, \tilde{\beta}$ и бозонных операторов $b_{i k}, b_{i k}^{+}$. Переставив операторы под знаком нормального произведения, тепловой гамильтониан (9) можно переписать в следуюшем виде:

$$
\mathcal{H}=: \sum_{1} \varepsilon_{1}\left(\alpha_{1}^{+} \alpha_{1}-\tilde{\alpha}_{1}^{+} \tilde{\alpha}_{1}\right)+\frac{1}{4} \sum_{1234} U_{1234}\left(\alpha_{1}^{+} \alpha_{3} \alpha_{2}^{+} \alpha_{4}-\tilde{\alpha}_{1}^{+} \tilde{\alpha}_{3} \tilde{\alpha}_{2}^{+} \tilde{\alpha}_{4}\right):
$$

В терминах операторов тепловых квазичастиц оператор $\alpha_{1}^{+} \alpha_{3}$ выглядит следующим образом:

$$
\alpha_{1}^{+} \alpha_{3}=x_{1} x_{3} \beta_{1}^{+} \beta_{3}+x_{1} y_{3} \beta_{1}^{+} \tilde{\beta}_{3}^{+}+y_{1} x_{3} \tilde{\beta}_{1} \beta_{3}+y_{1} y_{3} \tilde{\beta}_{1} \tilde{\beta}_{3}^{+} .
$$

Опустив в выражении для $\mathcal{H}$ члены, ответственные за взаимодействие коллективных возбуждений $\left(\sim \beta^{+} \beta \beta \beta\right.$ и $\left.\sim \beta^{+} \beta \beta^{+} \beta\right)$ [5], получим гармоническую часть теплового гамильтониана в виде

$$
\begin{aligned}
\mathcal{H} \simeq & : \sum_{1} \varepsilon_{1}\left(\beta_{1}^{+} \beta_{1}-\tilde{\beta}_{1}^{+} \tilde{\beta}_{1}\right)+ \\
& \left.+\frac{1}{4} \sum_{1234} U_{1234}\left(\left(x_{1} y_{3} \beta_{1}^{+} \tilde{\beta}_{3}^{+}+y_{1} x_{3} \tilde{\beta}_{1} \beta_{3}\right)\left(x_{2} y_{4} \beta_{2}^{+} \tilde{\beta}_{4}^{+}+y_{2} x_{4} \tilde{\beta}_{2} \beta_{4}\right)-\text { (t. c. }\right)\right):
\end{aligned}
$$

где (t. с.) означает "тильда-сопряженное" выражение.

Выразим теперь гамильтониан (П.1) через бозонные операторы $b_{i k}, b_{i k}^{+}$. Так как фермионные пары $\beta_{i}^{+} \beta_{i}$ и $\tilde{\beta}_{i}^{+} \tilde{\beta}_{i}$ удовлетворяют тем же самым коммуташионным соотношениям, что и операторы $\sum_{k} b_{i k}^{+} b_{i k}$ и $\sum_{k} b_{k i}^{+} b_{k i}$, последние можно считать их бозонными образами (в первом приближении).

Тогда для гамильтониана (П.1) получаем [10]

$$
\begin{aligned}
\mathcal{H}= & \sum_{12} \varepsilon_{12} b_{12}^{+} b_{12}+ \\
& +\frac{1}{4} \sum_{1234} U_{1234} \sqrt{1-q_{13}} \sqrt{1-q_{24}}\left[\left(x_{1} y_{3} b_{13}^{+}+y_{1} x_{3} b_{31}\right)\left(x_{2} y_{4} b_{24}^{+}+y_{2} x_{4} b_{42}\right)-\right. \\
& \left.\quad-\left(x_{1} y_{3} b_{31}^{+}+y_{1} x_{3} b_{13}\right)\left(x_{2} y_{4} b_{42}^{+}+y_{2} x_{4} b_{24}\right)\right]
\end{aligned}
$$

где

$$
\varepsilon_{12}=\varepsilon_{1}-\varepsilon_{2}
$$




\section{Список литературы}

[1] У. Умедзава, Х. Мацумото, М. Татики. Термополевая динамика и конденсированные состояния. М.: Мир, 1985.

[2] T. Hatsuda. Nucl. Phys. A. 1989. V. 492. P. 187.

[3] K. Tanabe. Phys. Rev. C. 1988. V. 37. P. 2802.

[4] O. Civitarese, A. I. DePaoli. Z. Phys. A. 1993. B. 344. S. 243.

[5] D.S. Kosov, A.I. Vdovin. Mod. Phys. Lett. A. 1994. V. 9. P. 1735.

[6] Н. Н. Боголюбов, Н. Н. Боголюбов (мл.). Введение в квантовую статистическую механику. М.: Наука, 1984.

[7] Ken-ji Hara. Progr. Theor. Phys. 1964. V. 32. P. 88.

[8] D. J. Rowe. Rev. Mod. Phys. 1968. V. 40. P. 1283.

[9] A. V. Avdeenkov, D. S. Kosov, A.I. Vdovin. Mod. Phys. Lett. A. 1996. V. 11. P. 853.

[10] M. Yamamura et. al.. Progr. Theor. Phys. 1990. V. 83. P. 749.

Поступила в редакцию 11.XI.1996 г.

\section{A. I. Vdovin, D.S. Kosov, W. Nawrocka \\ SELFCONSISTENT RANDOM PHASE APPROXIMATION FOR HOT FINITE FERMI-SYSTEMS}

By using a formalism of thermofield dynamics a new approach with the account of the influence of temperature and collective motion parameters on a mean field in hot finite Fermi-systems like atomic nuclei and metallic clusters is elaborated. Within the approach Pauli principle effects are considered in more correct way than in the standard termal random phase approximation. 